archives

of thermodynamics

Vol. 36(2015), No. 2, 3-25

DOI: $10.1515 /$ aoter-2015-0012

\title{
Numerical analysis of evaporation in microchannel under capillary pumping
}

\author{
JAROSEAW KARWACKI ${ }^{a}$ \\ HELENA NOWAKOWSKA ${ }^{a *}$ \\ MARCIN LACKOWSKI ${ }^{a}$ \\ DARIUSZ BUTRYMOWICZ ${ }^{b}$
}

a The Szewalski Institute of Fluid-Flow Machinery, Polish Academy of Sciences, Fiszera 14, 80-231 Gdańsk, Poland

${ }^{b}$ Bialystok University of Technology, Wiejska 45A, 15-351 Bialystok, Poland

\begin{abstract}
The paper presents numerical simulation of two-phase flow in a heated capillary with evaporation on the meniscus. To solve the problem, a model of evaporation from meniscus was developed in which the dynamics of liquid-vapour interface is described by the Cahn-Hilliard equation. The numerical simulations were performed using commercial software for 2D axially symmetric case. The flow evolution was analysed for different values of heat transfer coefficient at the capillary wall and inlet liquid mass flow rate.
\end{abstract}

Keywords: Capillary pumping; Evaporating meniscus; Microchannel; Cahn-Hilliard equation

\section{Nomenclature}

$\mathrm{C} \quad-$ constant, $\mathrm{m} / \mathrm{s}$

$C_{p} \quad-\quad$ specific heat capacity, $\mathrm{J} / \mathrm{kg} \mathrm{K}$

${ }^{*}$ Corresponding Author. E-mail: helena@imp.gda.pl 


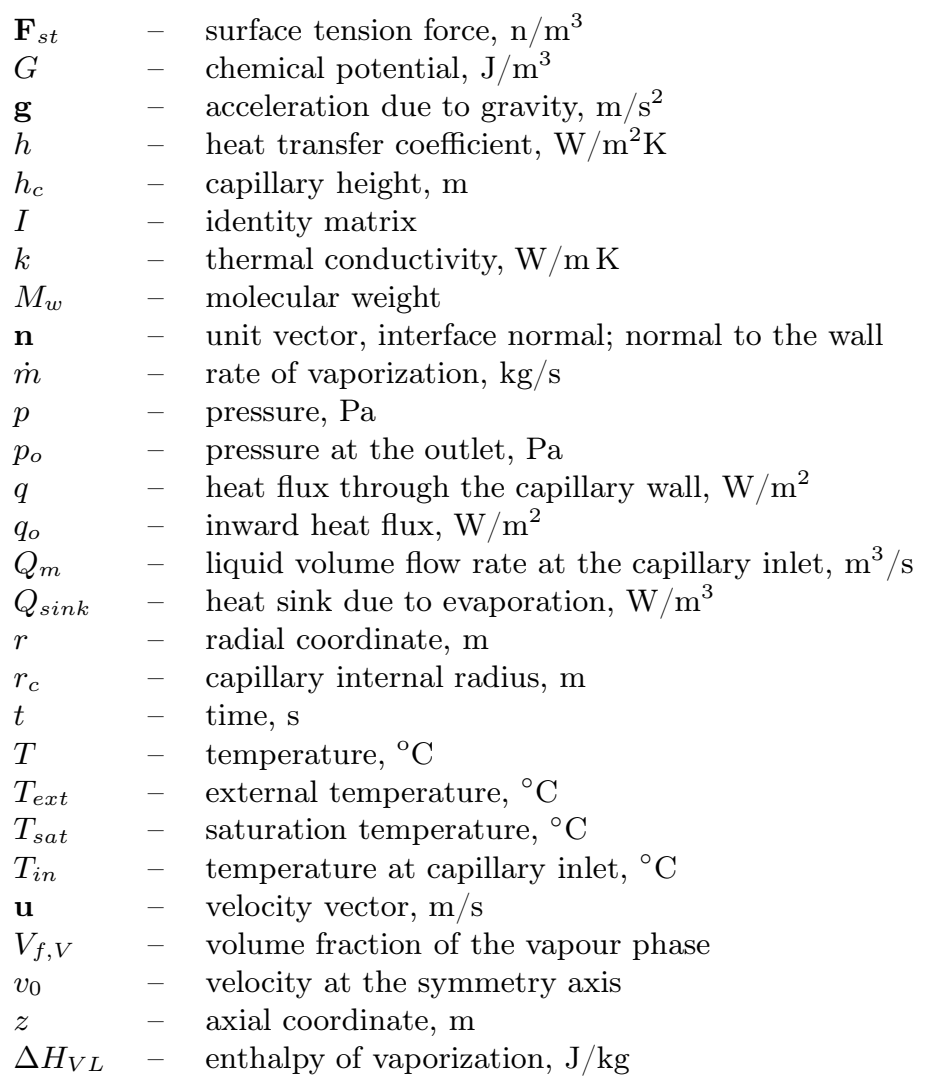

\section{Greek symbols}

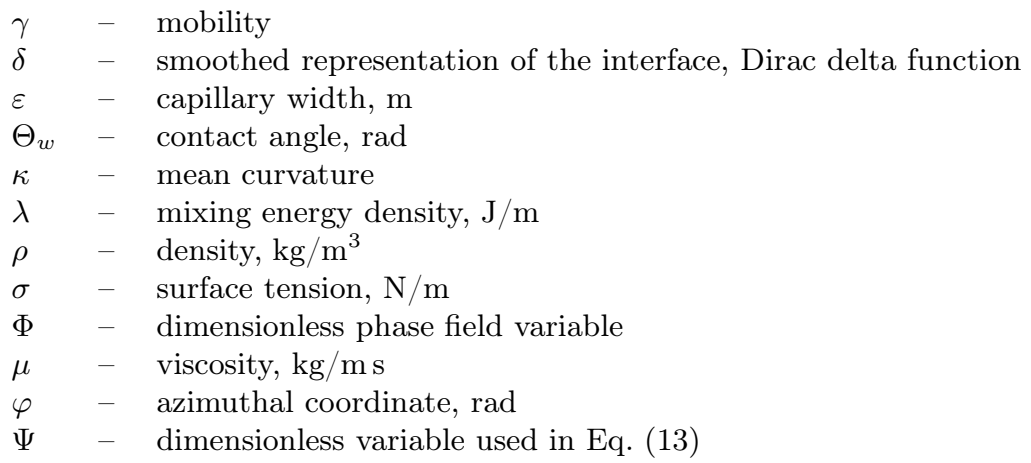


Subscripts

$L \quad-$ liquid

$V \quad-$ vapor

$T \quad-\quad$ transpose of matrix

\section{Introduction}

Application of microchannel heat exchangers in refrigeration and heat pump technologies has many advantages, such as small dimensions, substantial reduction of refrigerant charge in the system as well as possible enhancement of heat transfer. The main drawback of this type of exchangers is significant flow resistance. However, it can be substantially reduced by the capillary pumping effect $[1,2]$.

The goal of this work was to perform a theoretical analysis of water flow in a heated capillary. This problem includes the following main issues: twophase flow, meniscus forming and the phase change. To achieve the goal, a model of evaporation from meniscus has been developed and 2D axially symmetric numerical simulations have been performed using commercial software - COMSOL Multiphysics. The position of the meniscus, fluid temperature and pressure distribution in dependence of the heat flux and mass flow have been found for a capillary of given dimensions. Influence of liquid superheating on the results has also been analysed.

\section{Model of two-phase flow with phase change in a heated capillary}

\subsection{Problem statement}

The problem to be modeled can be defined as follows. The glass capillary with the radius, $r_{c}$, and height, $h_{c}$, is placed vertically. Water is delivered to the capillary through the inlet at the bottom with the mass flow rate $Q_{m}$. The capillary is heated through the walls with the heat flux $Q_{h}$, so the water is heated and its temperature increases up to the saturation temperature $T_{\text {sat }}$ and evaporates. In the experiment, the heat flux is adjusted to the mass flow in such way that - in the stationary state - the height of the water column reaches about 0.5 of the capillary height. The pressure at the capillary outlet is atmospheric. The capillary is heated using hot air of the temperature $T_{\text {ext }}$, which is higher than the saturation temperature. This method of heating causes that the fluid temperature cannot exceed 
the heating air temperature. Both capillary and gravitational forces have to be taken into account.

As far as physics is concerned, the evaporation (at saturation temperature) from a capillary is different from pool boiling, so typical boiling curve cannot be applied. Such evaporation can be regarded as a metastable process without bubbles forming. The temperature on the interface separating liquid and vapour is assumed to be equal to the saturation temperature.

\subsection{Governing equations}

The whole theory and equations presented in Subsections 2.2, 2.3 and 2.4 are based on COMSOL Multiphysics documentation [3,4]. The problem can be solved using proper set of heat and momentum equations, from which the temperature, pressure and velocities can be found.

The velocity field and pressure for the liquid phase of a fluid are described by the incompressible Navier-Stokes equations

$$
\begin{aligned}
& \rho_{L} \frac{\partial \mathbf{u}_{L}}{\partial t}+\rho_{L}\left(\mathbf{u}_{L} \cdot \nabla\right) \mathbf{u}_{L}= \\
& =\nabla\left[-p_{L} \mathbf{I}+\mu_{L}\left(\nabla \cdot \mathbf{u}_{L}+\left(\nabla \cdot \mathbf{u}_{L}\right)^{T}\right)\right]+\rho_{L} \mathbf{g}+\mathbf{F}_{s t}, \\
& \nabla\left(\mathbf{u}_{L}\right)=0
\end{aligned}
$$

where $\rho_{L}$ and $\mathbf{u}_{L}$ are the fluid density and velocity, respectively $p_{L}$ is the pressure, $\mu_{L}$ is the viscosity and $\mathbf{I}$ is the identity matrix, and the superscript $T$ denotes the transpose. Subscript $L$ denotes liquid phase. $\mathbf{F}_{s t}$ is the surface tension force acting at the liquid-vapour interface and is calculated from:

$$
\mathbf{F}_{s t}=\nabla \cdot \mathbf{T}
$$

with

$$
\mathbf{T}=\sigma\left(\mathbf{I}-\left(\mathbf{n n}^{T}\right)\right) \delta,
$$

where $\mathbf{n}$ is the interface normal, $\sigma$ is the surface tension coefficient, and $\delta$ is a Dirac delta function that is nonzero only at the fluid interface.

The velocity field and pressure for the vapour phase of a liquid are described by the compressible Navier-Stokes equations

$$
\begin{aligned}
& \rho_{V} \frac{\partial \mathbf{u}_{V}}{\partial t}+\rho_{V}\left(\mathbf{u}_{V} \cdot \nabla\right) \mathbf{u}_{V}= \\
& \quad=\nabla\left[-p_{V} \mathbf{I}+\mu_{V}\left(\nabla \cdot \mathbf{u}_{V}+\left(\nabla \cdot \mathbf{u}_{V}\right)^{T}\right)-\frac{2}{3} \mu_{V}\left(\nabla \cdot \mathbf{u}_{V}\right) \mathbf{I}\right]+\rho_{V} \mathbf{g},(5)
\end{aligned}
$$




$$
\frac{\partial}{\partial t} \rho_{V}+\nabla\left(\rho_{V} \mathbf{u}_{V}\right)=0,
$$

where physical quantities are designed by the same letters that in Eq. (1) with the subscript $V$ denoting the vapour phase, and $\mathbf{g}$ is the acceleration due to gravity. The heat equations are solved independently for the vapour and liquid phase:

$$
\begin{gathered}
\rho_{V} C_{p, V} \frac{\partial T_{V}}{\partial t}+\rho_{V} C_{p, V}\left(\mathbf{u}_{V} \cdot \nabla\right) T_{V}=\nabla \cdot\left(k_{V} \nabla t_{V}\right), \\
\rho_{L} C_{p, L} \frac{\partial T_{L}}{\partial t}+\rho_{L} C_{p, L}\left(\mathbf{u}_{L} \cdot \nabla\right) T_{L}=\nabla \cdot\left(k_{L} \nabla t_{L}\right),
\end{gathered}
$$

where $C_{p}$ is the specific heat capacity, and $k$ is the thermal conductivity.

\subsection{Boundary conditions for the interface}

The interface velocity, liquid velocity and vapour velocity are connected in the following way:

$$
\mathbf{u}_{\text {int }}=\mathbf{u}_{L}-\frac{\dot{m}}{\rho_{L}} \mathbf{n} .
$$

The natural boundary condition on the interface for the vapour phase is

$$
\mathbf{n} \cdot \rho_{V} \mathbf{u}_{V}=\dot{m}\left(-\frac{\rho_{V}}{\rho_{L}}\right)+\left(\mathbf{n} \cdot \rho_{V} \mathbf{u}_{L}\right) .
$$

The natural boundary condition on the interface for the liquid phase is

$$
\begin{aligned}
& \mathbf{n}\left[-\rho_{L} \mathbf{I}+\mu_{L}\left(\nabla \cdot \mathbf{u}_{L}+\left(\nabla \cdot \mathbf{u}_{L}\right)^{T}\right)\right]= \\
& \quad=\dot{m}\left(\mathbf{u}_{\mathbf{L}}-\mathbf{u}_{V}\right)+\sigma \kappa \mathbf{n}+\mathbf{n}\left[-p_{V} \mathbf{I}+\mu_{V}\left(\nabla \cdot \mathbf{u}_{V}+\left(\nabla \cdot \mathbf{u}_{V}\right)^{T}\right)\right],
\end{aligned}
$$

which results from the force balance on the interface. The first term on the right hand side represents a reaction force due to acceleration of vapour away from the liquid surface. The second term is the surface tension and the last term on the right hand side is the sum of pressure and viscous forces acting on the liquid from the vapour. The mass flux leaving the liquid surface leads to an increase in pressure of the vapour. The pressure exerts a force on the liquid surface and the vapour region begins to expand. The presence of the surface tension force leads to a discontinuity in pressure across the interface. The mean curvature is designed by $\kappa$. In the energy equation, the 
temperature at the interface is fixed at the saturation temperature, which may be a function of pressure

$$
T=T_{\text {sat }}(p) .
$$

The mass flux leaving the interface can then be evaluated from the conductive heat flux:

$$
\dot{m}=-\left(\frac{M_{w}}{\Delta H_{V L}}\right) \mathbf{n} \cdot k_{V} \nabla T_{V},
$$

where $k$ is the thermal conductivity, $M_{w}$ is the molecular weight of the medium, $\Delta H_{V L}$ is the enthalpy of vaporization, and $\dot{m}$ is the rate of vaporization. This approximation is obtained by neglecting the kinetic energy and work due to viscous forces [5].

Equations (1)-(7) along with boundary conditions Eqs. (8)-(12) represent a complete description of the evaporating flow.

\subsection{Solution of the problem in COMSOL Multiphysics}

The problem presented above was solved with COMSOL Multiphysics software using a method, where the equations governing the interface dynamics of a two-phase flow are described by the Cahn-Hilliard equation for the so called phase field variable. To allow for the change of phase the equation for the phase field variable should be modified, as it is shown in [6]. The modified Cahn-Hilliard equation has the following form:

$$
\frac{\partial \Phi}{\partial t}+\mathbf{u} \cdot \nabla \Phi-\dot{m} \delta\left(\frac{V_{f, V}}{\rho_{V}}+\frac{V_{f, L}}{\rho_{L}}\right)=\nabla \frac{\gamma \lambda}{\varepsilon^{2}} \nabla \Psi,
$$

where $\Phi$ is the dimensionless phase field variable such that $-1 \leq \Phi \leq 1$. The volume fraction of the vapour phase is $V_{f, V}$, and the volume fraction of the liquid is $V_{f, L}$. They are connected via the formula $V_{f, V}=1-V_{f, L}$. The quantity $\lambda$ is the mixing energy density and $\varepsilon$ is the capillary width that scales with the thickness of the interface. These two parameters are related to the surface tension coefficient by the formula

$$
\sigma=\frac{2 \sqrt{2}}{3} \frac{\lambda}{\varepsilon} .
$$

Quantity $\gamma$ in Eq. (1) is the interface mobility, which determines the time scale of the Cahn-Hilliard diffusion and must be large enough to retain a constant interfacial thickness but small enough so that the convective terms 
are not too excessively damped. The equation governing dimensionless variable $\Psi$ appearing in Eq. (13) is

$$
\Psi=-\nabla \varepsilon^{2} \nabla \Phi+\left(\Phi^{2}-1\right) \Phi .
$$

The quantity $\delta$ is a smoothed representation of the interface between the two phases. It is defined as

$$
\delta=6 V_{f}\left(1-V_{f}\right) \frac{|\nabla \Phi|}{2} .
$$

The momentum equation includes surface tension effects as a volumetric body force

$$
\rho \frac{\partial \mathbf{u}}{\partial t}+\rho(\mathbf{u} \cdot \nabla) \mathbf{u}=\nabla \cdot\left[-p_{L} \mathbf{I}+\mu\left(\nabla \cdot \mathbf{u}+(\nabla \cdot \mathbf{u})^{T}\right)\right]+\rho \mathbf{g}+G \nabla \Phi,
$$

where $G$ is the chemical potential. The continuity equation is modified to account for the phase change from liquid to vapour:

$$
\nabla \cdot \mathbf{u}=\dot{m} \delta\left(\frac{1}{\rho_{V}}-\frac{1}{\rho_{L}}\right)
$$

The problem is however with the definition of the rate of phase change. Equation (12) cannot be used because the peak temperature gradient does not coincide with the interface. This leads to a substantial underestimate of the mass flux leaving the surface. Instead, the mass flux can be approximated by the following expression

$$
\dot{m}=-\left(\frac{M_{w}}{\Delta H_{V L}}\right) \mathbf{n} \kappa_{V} \nabla T_{V}=C \rho_{L} \frac{T-T_{s a t}}{T_{\text {sat }}},
$$

where $C$ is a constant. This expression is analogous to specifying a heat transfer coefficient on the external boundary of the heat transfer problem. The mass flux appears in energy equation as

$$
\rho C_{p} \frac{\partial T}{\partial t}+\rho C_{p}(\mathbf{u} \cdot \nabla) T=\nabla(k \nabla T)-\frac{\dot{m} \delta \Delta H_{V L}}{M_{w}} .
$$

The last term in Eq. (20) represents the heat sink due to evaporation, $Q_{\text {sink }}$. The combination of Eqs. (19) and (20) naturally forces the interface temperature to the saturation temperature. The constant $C$ should be large enough that the temperature at the interface remains at the saturation 
temperature but not so large to induce numerical instabilities. The thermal conductivity and specific heat capacity are computed as functions of the volume fraction of two phases:

$$
\begin{gathered}
\kappa=\left(\kappa_{L}-\kappa_{V}\right) V_{f, L}+\kappa_{V}, \\
C_{p}=\left(C_{p, L}-C_{p, V}\right) V_{f, L}+C_{p, V} .
\end{gathered}
$$

\subsection{Boundary conditions}

Due to the axial symmetry of the analysed model all calculations were performed in cylindrical coordinate system $(r, z, \varphi)$, where $r, z$ and $\varphi$ represent the radial, axial and azimuthal coordinate, respectively. The domain used for calculations and the directions of the coordinates are shown in Fig. 1. Point $\mathrm{A}$ is assumed in the centre of the coordinate system $(r=0, z=0)$. The imposed boundary conditions (BCs) are presented in Tab. 1.

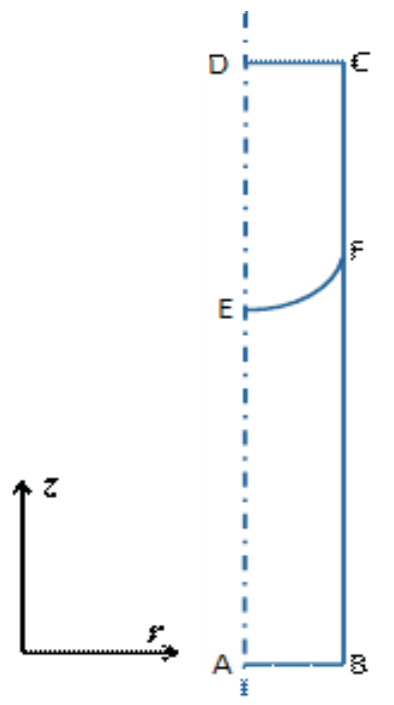

Figure 1: The calculation domain.

The symmetry axis boundary conditions require that all partial derivatives over radial coordinate ( $r$-coordinate) are equal to zero. By imposing the laminar inflow boundary condition it is assumed that a fully developed laminar flow is at the inlet.

The wetted wall boundary condition is imposed on walls in contact with 
Table 1: Boundary conditions (boundary description as in Fig. 1).

\begin{tabular}{|l|l|l|}
\hline Boundary & \multicolumn{1}{|c|}{ Flow } & \multicolumn{1}{c|}{ Heat } \\
\hline \hline AD & Symmetry axis & Symmetry axis \\
\hline AB (inlet) & Laminar inflow, mass flow rate $Q_{m}$ & $T=T_{i n}$ \\
\hline DC (outlet) & $p_{0}=101325 \mathrm{~Pa}$, no vicious stress & Conductive heat flux $=0$ \\
\hline BC (wall) & Wetted wall, contact angle $=\theta_{w}$ & Inward heat flux $=q_{0}$ \\
\hline
\end{tabular}

the fluid-fluid interface. If this boundary condition is used, the fluid-fluid interface can move along the wall. For the phase field method, the motion of the interface on the boundary due to advection is zero and so the no slip boundary condition $(\mathbf{u}=0)$ is used in the momentum equation. The following boundary condition defines the contact angle between liquid and the wall

$$
\mathbf{n} \cdot \varepsilon^{2} \nabla \Phi=\varepsilon^{2} \tan \left(\pi / 2-\theta_{w}\right)|\nabla \Phi-(\mathbf{n} \cdot \nabla \Phi)| \mathbf{n},
$$

where $\theta_{w}$ is the user-defined contact angle. The phase field help variable is assigned to the boundary condition

$$
\mathbf{n} \cdot \frac{\gamma \lambda}{\varepsilon^{2}} \nabla \psi=0 .
$$

The inward heat flux $q_{0}$ is imposed in the following form:

$$
q_{0}=h\left(T_{e x t}-T\right),
$$

where $h$ is the heat transfer coefficient and $T_{e x t}$ is the external temperature. This form of heat boundary condition is adequate when the capillary is heated by hot air flow. It is taken that only the convective heat flow is on the capillary outlet (the conductive heat flow is equal to zero).

\section{Results}

\subsection{Input data}

The calculations were performed for the capillary radius $r_{c}=0.1 \mathrm{~mm}$ and the height $h_{c}=20 \mathrm{~mm}$. The two fluids considered were water and water vapour. The liquid density was taken as $\rho_{L}=1000 \mathrm{~kg} / \mathrm{m}^{3}$, and the viscosity as $\mu_{L}=1 \times 10^{3} \mathrm{Pas}$. The vapour density was given by the ideal gas law, and the viscosity of $\mu_{V}=4 \times 10^{5} \mathrm{Pas}$ was specified. This is slightly higher than 
the actual viscosity of water vapour but leads to a more stable solution. The thermal conductivity of liquid was taken as $k_{L}=0.63 \mathrm{~W} /(\mathrm{mK})$ and of vapour was calculated from

$$
k_{V}=8.351 \times 10^{-5}\left[\mathrm{~W} /(\mathrm{m} \mathrm{K})^{2}\right] T-7.4556 \times 10^{-3}[\mathrm{~W} /(\mathrm{m} \mathrm{K})] .
$$

The pressure in the surrounding environment was assumed as $p_{0}=101325 \mathrm{~Pa}$ and the saturation temperature was taken as $T_{\text {sat }}=100{ }^{\circ} \mathrm{C}$. The surface tension coefficient was taken as $\sigma=0.0588 \mathrm{~N} / \mathrm{m}$. The contact angle was chosen as $\theta_{w}=67^{\circ}$. According to the experimental conditions, the external temperature was set as $T_{e x t}=150{ }^{\circ} \mathrm{C}$ and the inlet temperature $T_{\text {in }}=70{ }^{\circ} \mathrm{C}$. The calculations were performed for different values of the heat transfer coefficient, $h$, and the liquid volume flow rate, $Q_{m}$. The constant $C$ in Eq. (19) was set as $0.03 \mathrm{~m} / \mathrm{s}$ and it was confirmed that increasing or decreasing this value by factor of two does not change the results. The quantity $\varepsilon$ in Eq. (13) was chosen as $5 \times 10^{-5} \mathrm{~m}$. It was confirmed in preliminary calculations that such value ensures that the interface region is narrow enough and may be estimated as $0.2 \mathrm{~mm}$. For larger values of $\varepsilon$ the interface region is too fuzzy.

The calculations were performed in a time dependent form. The initial conditions were taken as follows. The temperature was set to $T_{i n}$ in the whole region, the pressure equal to $p_{0}$ and the velocity having only radially parabolic axial component $v_{z}$, which responds to a laminar flow

$$
v_{z}(r)=v_{0}\left(1-\left(r / r_{c}\right)^{2}\right),
$$

where $v_{0}$ is the velocity at the symmetry axis and is adjusted to the volume flow rate at the inlet.

The liquid-vapour interface was assumed as horizontal $(z=$ const $)$ and its position was in the middle of the capillary height $z=10 \mathrm{~mm}$.

\subsection{Time development of the flow}

First calculations were performed for liquid volume flow rate $Q_{m}=0.7$ $\mu \mathrm{L} / \mathrm{min}$, assuming that the heat transfer coefficient $h$ is constant and equal to $17.5 \mathrm{~W} /\left(\mathrm{m}^{2} \mathrm{~K}\right)$. Figure 2 shows the volume fraction of the vapour phase in four time moments in a part of the calculation domain presented in Fig. 1, which is in the vicinity of the meniscus. In both figures there is the same coordinate system and values of $\mathrm{r}$ and $\mathrm{z}$ (horizontal and vertical coordinates, respectively) seen in Fig. 2 are given in millimeters. The region where there 
is only water is marked blue $\left(V_{f, V}=0\right)$ and where there is only vapour is marked brown $\left(V_{f, V}=1\right)$ The additional black lines show $0.4,0.5$ and 0.6 levels of $V_{f, V}$. It is seen that meniscus is formed almost immediately, which means that although the initial condition for the water-vapour interface was chosen as $z=$ const $=0 \mathrm{~mm}$, consistency of boundary conditions require that the meniscus is formed as early as $t=0 \mathrm{~s}$. From Fig. 2 it is seen that the meniscus moves up by about $0.3 \mathrm{~mm}$ within $0.1 \mathrm{~s}$.

(a)

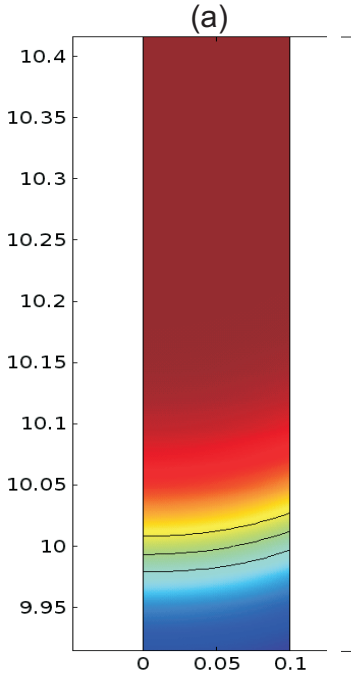

(b)

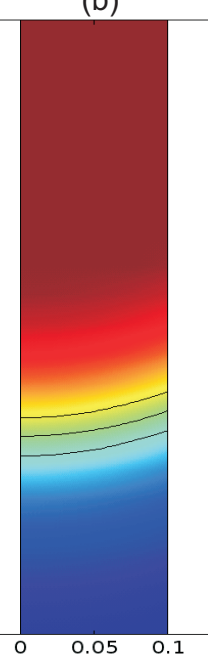

(c)

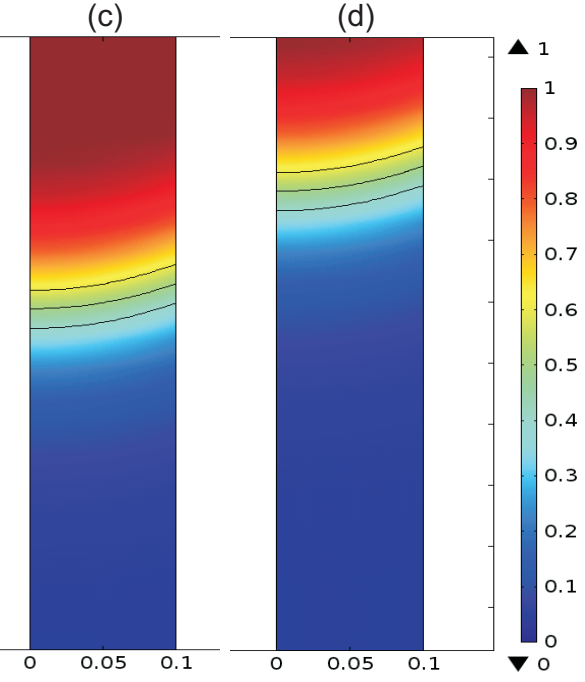

Figure 2: Spatial distributions of the volume fraction of the vapour phase, $V_{f, V}, Q_{m}=$ $0.7 \mu \mathrm{L} / \mathrm{min}, h=17.5 \mathrm{~W} /\left(\mathrm{m}^{2} \mathrm{~K}\right):$ (a) $t=0 \mathrm{~s}$, (b) $t=0.01 \mathrm{~s}$, (c) $t=0.05 \mathrm{~s}$, (d) $t=0.1 \mathrm{~s}$. Coordinate system is the same as in Fig. 1. Numbers on the axis represent the coordinate dimensions in $\mathrm{mm}$.

Figure 3 shows the volume fraction of the vapour phase for six time moments covering longer interval than that in Fig. 2. Values of $z$ and $r$ coordinates (in millimetres) are shown only in Fig. 3(a). The scales of the coordinates are different to enable to observe the results in the whole calculation domain. It is seen that the position of the interface is $10 \mathrm{~mm}$ at the beginning and next it moves up reaching about $11.5 \mathrm{~mm}$ for $t=2 \mathrm{~s}$. After $3 \mathrm{~s}$ it starts moving down and after $5 \mathrm{~s}$ it is almost at $10 \mathrm{~mm}$ level again. This effect is also seen in Fig. 4, where the volume fraction is shown of the vapour phase $V_{f, V}$ at the capillary wall for several time moments. The effect of the moving up is the consequence of the fact that the imposed flow at the inlet and the capillary forces prevail the effect of the gravitational forces. When the temperature increases and evaporation of the liquid is 


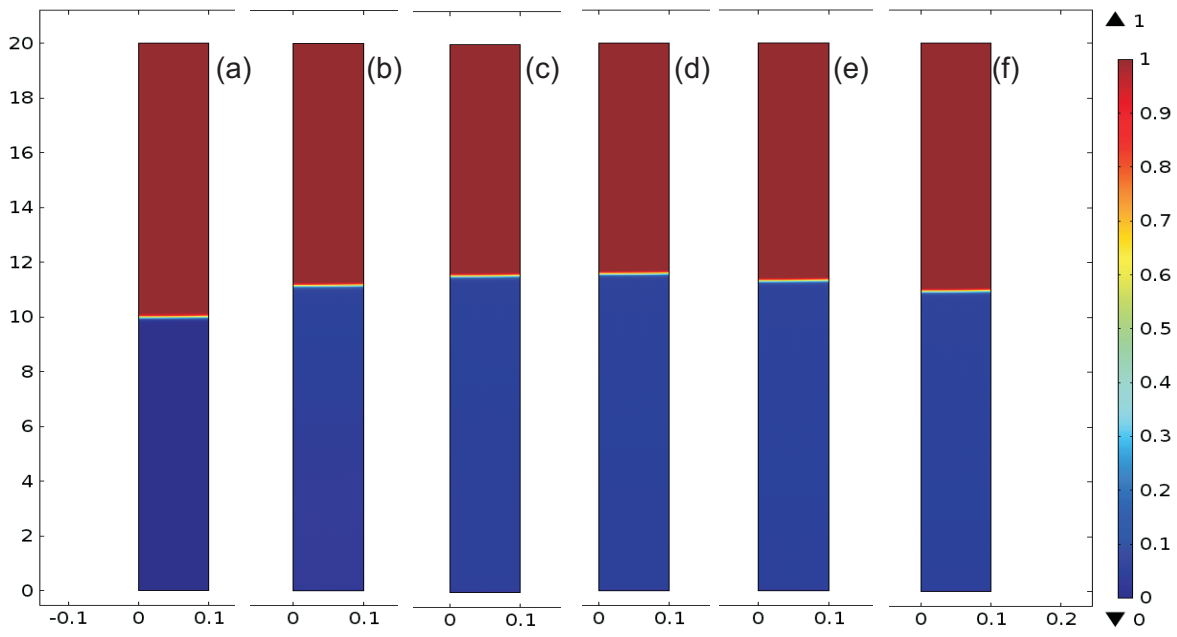

Figure 3: Spatial distributions of the volume fraction of the vapour phase $V_{f, V}, Q_{m}=$ $0.7 \mu \mathrm{L} / \mathrm{min}, h=17.5 \mathrm{~W} /\left(\mathrm{m}^{2} \mathrm{~K}\right):$ (a) $t=0 \mathrm{~s}$, (b) $t=1 \mathrm{~s}$, (c) $t=2 \mathrm{~s}$, (d) $t=3 \mathrm{~s}$, (e) $t=4 \mathrm{~s}$, (f) $t=5 \mathrm{~s}$. Coordinate system is the same as in Fig. 1. Numbers on the axis represent the coordinate dimensions in $\mathrm{mm}, r$ - and $z$-coordinate scale are different.

strong enough the downward movement occurs.

Figure 5 shows the temperature distributions for the same six time moments as that shown in Fig. 3. The additional, almost horizontal line shows $V_{f, V}=0.5$ contour, i.e. the position of the meniscus. It is seen that the temperature increases from the initial $70^{\circ} \mathrm{C}$ quickly in the vapour phase. It reaches the external temperature of $150^{\circ} \mathrm{C}$ after $1 \mathrm{~s}$. The temperature of the liquid phase increases slowly. From Fig. 6, showing the temperature at the capillary wall, it is seen that the liquid temperature exceeds the saturation temperature $\left(100^{\circ} \mathrm{C}\right)$ after about $5 \mathrm{~s}$.

Figure 7 presents the distribution of $Q_{\text {sink }}$, heat sink term defined in Eq. (20). It is greater than zero when the fluid temperature is greater than saturation temperature in the region of the phase change (liquid-vapour) interface. This is the condition of evaporation from the meniscus. Diagram reveals that the evaporation is limited to the phase interface region. It starts at about $t=1 \mathrm{~s}$ and the region of evaporation is close to the capillary tube. As the capillary is heated the evaporation region extends and covers the whole radius of the interface. It becomes strong enough that the effect overcomes the induced flow and the meniscus moves down, reaching the primary level after about $5 \mathrm{~s}$. This effect is also shown in Fig. 8, where the 


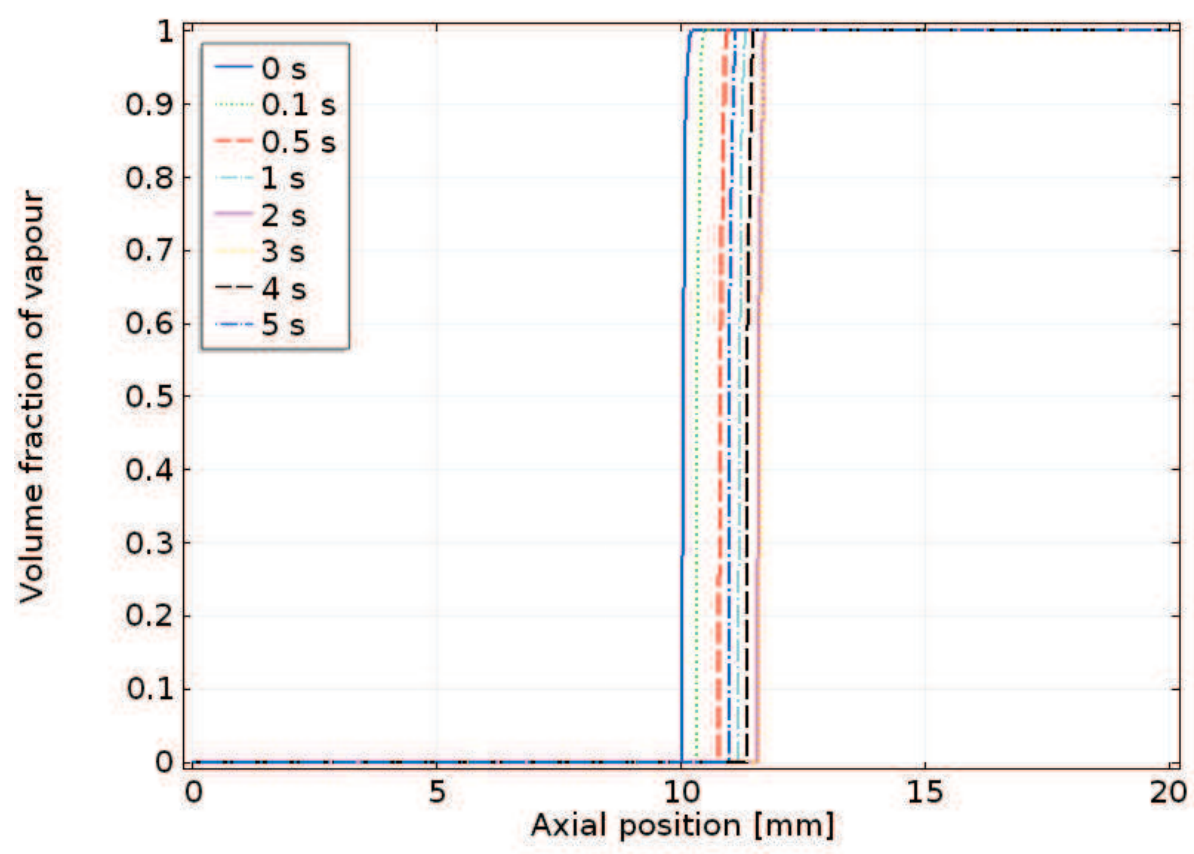

Figure 4: The volume fraction of the vapour phase, $V_{f, V}$, at the capillary wall.

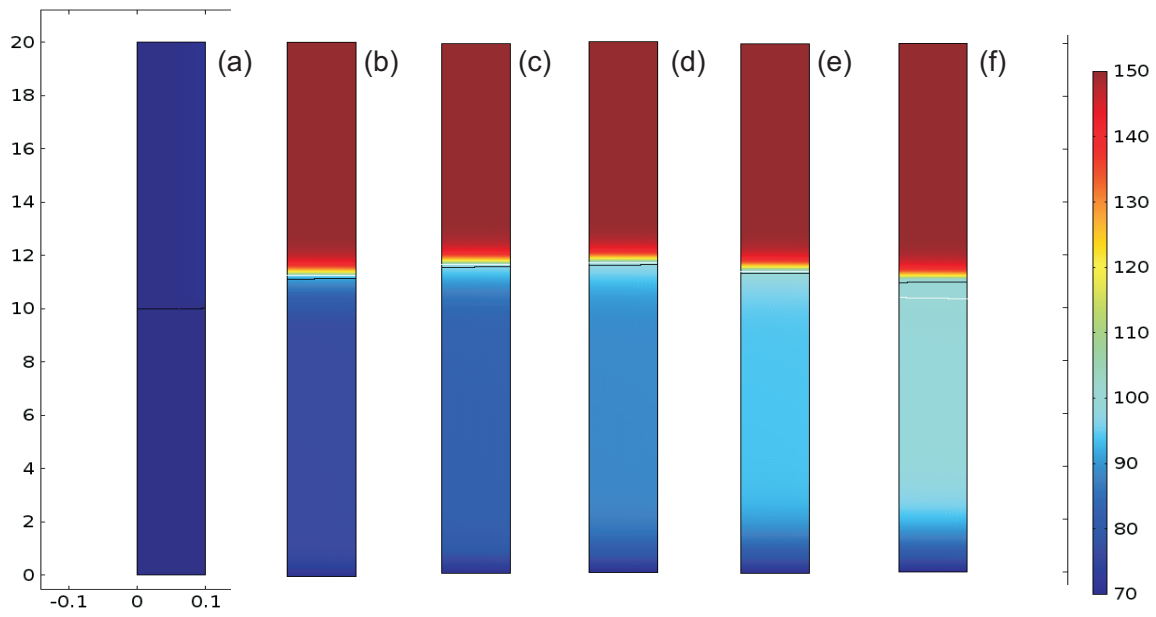

Figure 5: Spatial distributions of the heat $\operatorname{sink} Q_{\operatorname{sink}}$; (a) $t=0 \mathrm{~s}$, (b) $t=1 \mathrm{~s}$, (c) $t=2 \mathrm{~s}$, (d) $t=3 \mathrm{~s}$, (e) $t=4 \mathrm{~s}$, (f) $t=5 \mathrm{~s}$. The temperature scale at the right is in ${ }^{\circ} \mathrm{C}$. 


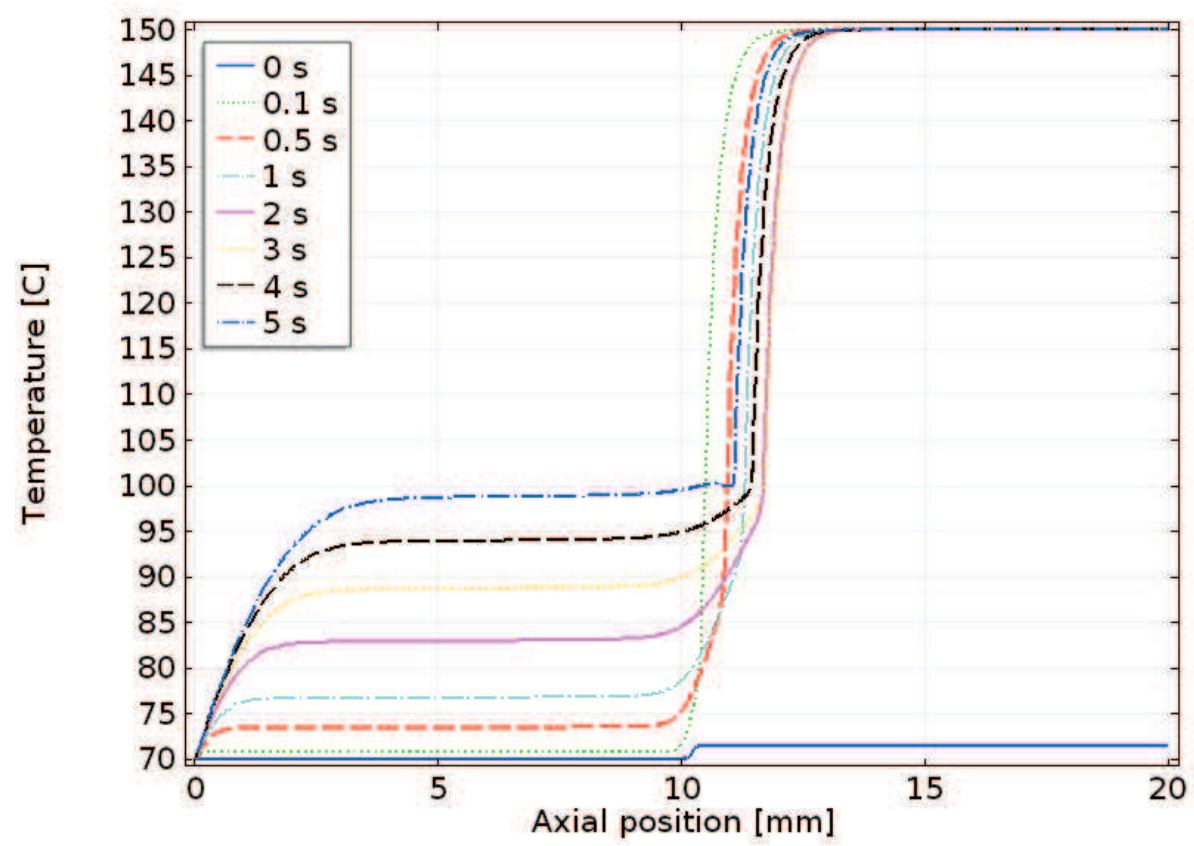

Figure 6: The temperature at the capillary wall in several time instants.

heat sink at the capillary wall is presented. For $t<1 \mathrm{~s}$ the heat sink is zero, it becomes noticeable for $t \cong 1$ (maximal value is below $106 \mathrm{~W} / \mathrm{m}^{3}$ ). With time both the maximal value of the heat sink and the width of the evaporation layer increase causing that the evaporation of water increases and the position of the meniscus moves down.

In Fig. 9, supplementary information is given. It shows how the fluid pressure at the capillary wall changes. The pressure at the outlet is assumed to be zero. A drop of the pressure corresponding to the capillary pressure is seen in the phase interface region. For all cases it is almost equal to its equilibrium value $459 \mathrm{~Pa}$, which can be found from $\Delta p=2 \sigma \cos \left(\theta_{w}\right) / r_{c}$.

\subsection{Influence of the heat transfer coefficient}

In the next step of analysis, the influence of heating conditions on the processes has been analysed. A parametric study with four values of the heat transfer coefficient, $h$, has been performed. According to Eq. (25), increasing $h$ causes increasing the inward heat flux $q_{0}$. The results are presented in 

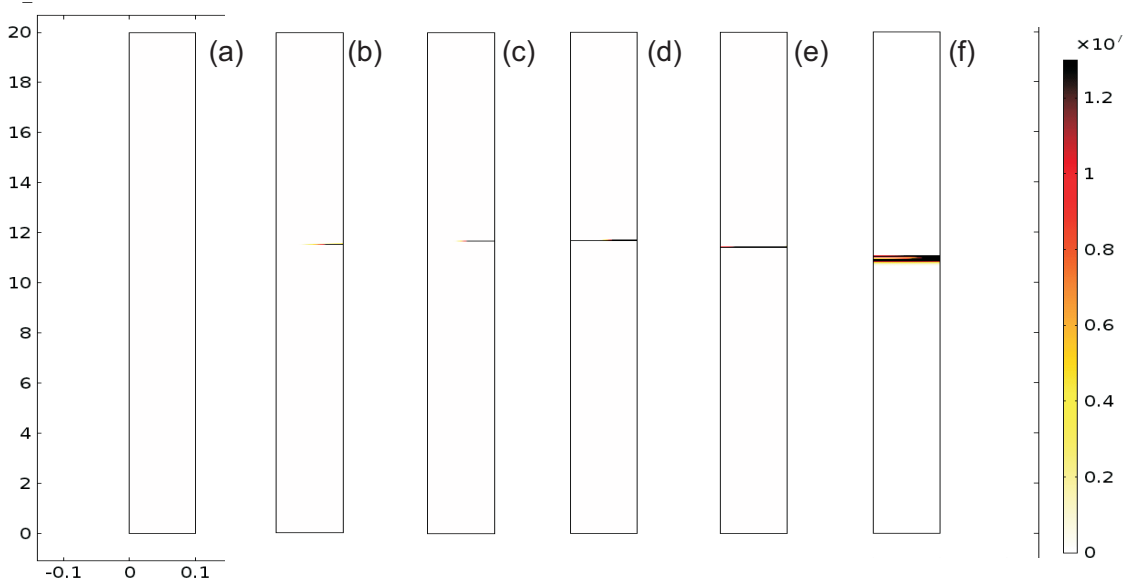

Figure 7: The heat $\operatorname{sink} Q_{\sin k}$; (a) $t=0 \mathrm{~s}$, (b) $t=1 \mathrm{~s}$, (c) $t=2 \mathrm{~s}$, (d) $t=3 \mathrm{~s}$, (e) $t=4 \mathrm{~s}$, (f) $t=5 \mathrm{~s}$. The heat sink scale at right is in $\mathrm{W} / \mathrm{m}^{3}$.

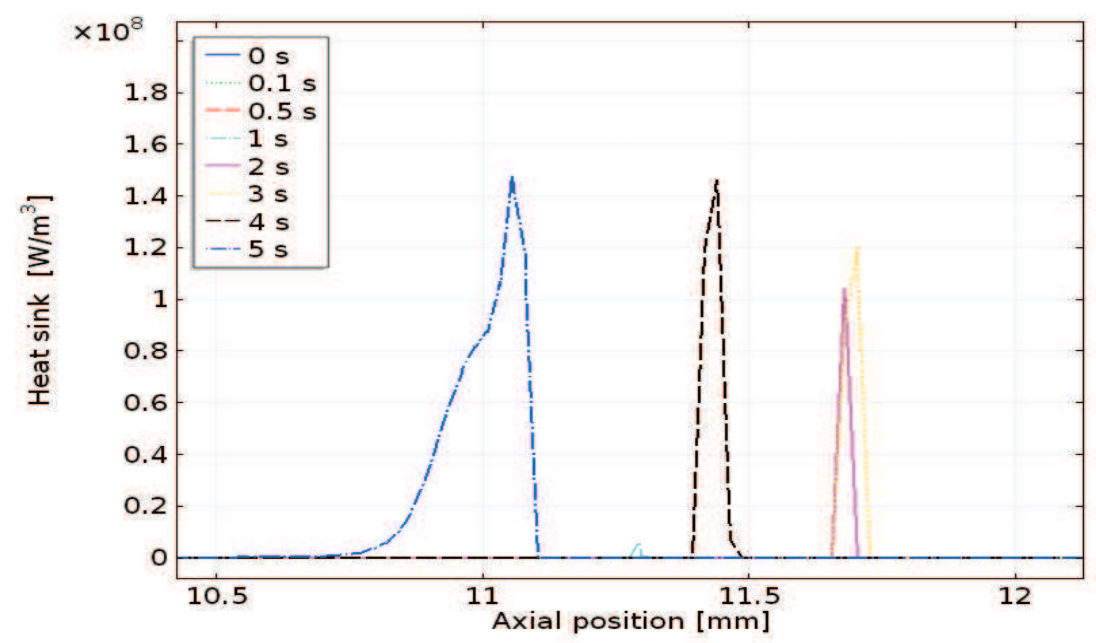

Figure 8: The heat $\operatorname{sink} Q_{\text {sink }}$ at the capillary wall in several time instants.

Figs. 10-13. From Figs. 10 and 11 it is seen that for the same time moment the higher the heat flux, the lower the position of the interface, and higher temperature. It is accordance with expectations because stronger heating causes the temperature increases quicker and the evaporation is stronger, which causes that the position of the interface moves down. It is shown in Fig. 12 how heat sink term depends on the coefficient $h$. For the same time 


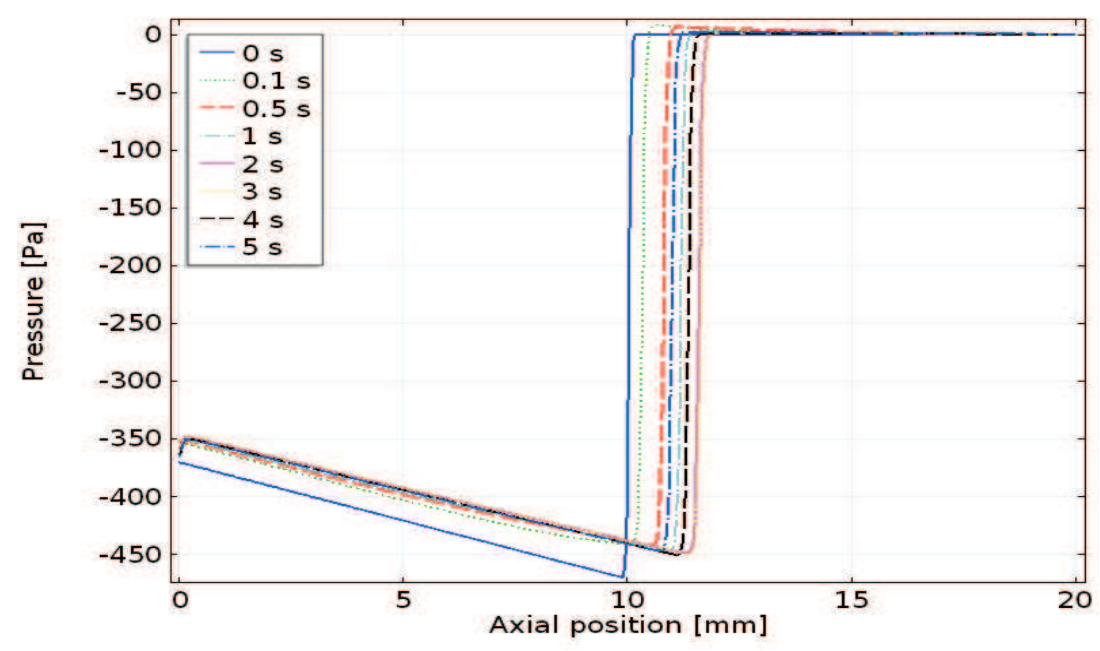

Figure 9: The pressure at the capillary wall in several time instants.

moment the heat sink term increases with the coefficient $h$. The position of the maximal heat sink term moves down with increasing $h$.

Figure 13 presents how the total heat flux penetrating the capillary walls changes with time. It is seen that in every time moment the higher the coefficient $h$, the higher the heat flux. During first milliseconds the heat flux is larger than $1 \mathrm{~kW} / \mathrm{m}^{2}$ which is connected with heating vapour. When the vapour reaches temperature about $150{ }^{\circ} \mathrm{C}$, the heat flux almost stabilizes for the first $5 \mathrm{~s}$.

\subsection{Influence of the mass flow rate}

In the following step of analysis, the influence of mass flow rate, $Q_{m}$, on the processes has been analysed. A parametric study with three values of the inlet volume flow rate $(0.35,0.7$, and $1 \mu \mathrm{L} / \mathrm{min})$ has been performed. Figures 14-16 present results of the calculations. It is seen from Fig. 14 that - as it is expected - for bigger mass flow rate the maximal value of the position along $z$-axis is bigger and the time after which the maximum is achieved is longer.

Figures 15 and 16 present influence of the mass flow rate on the temperature of the capillary wall. It is seen that the difference between the cases, which is seen in the vicinity of the inlet and close to the interface, increases with time. 

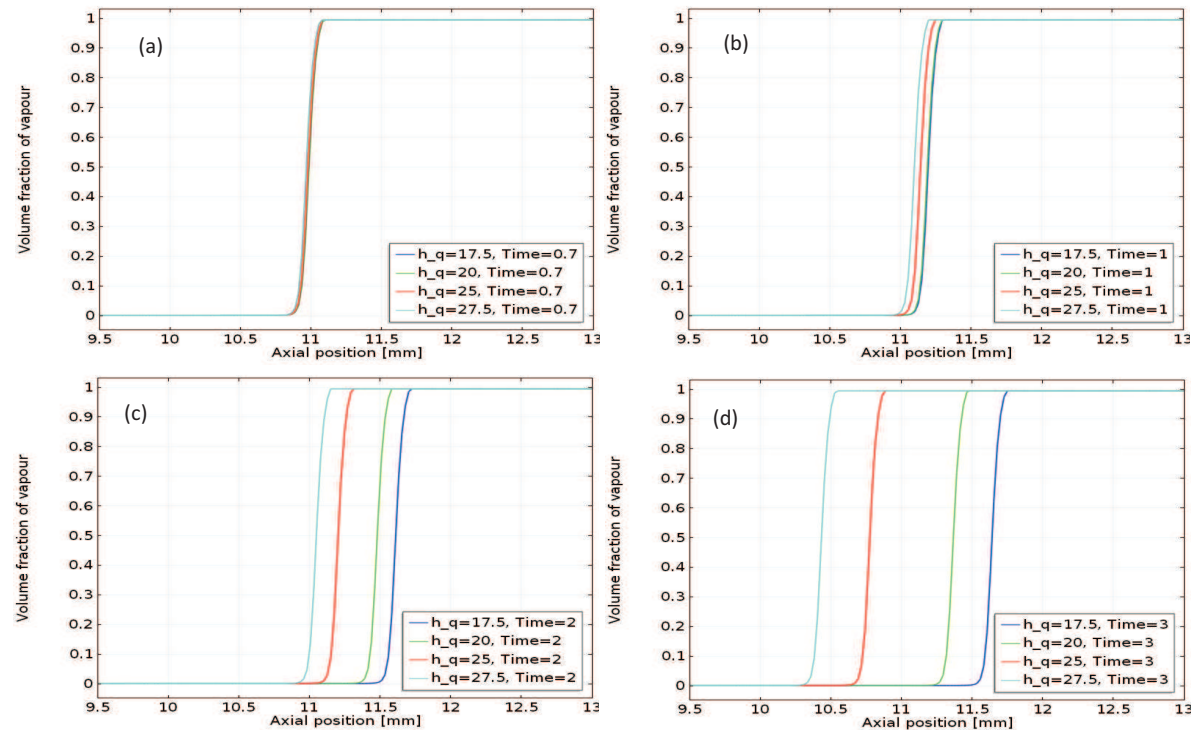

Figure 10: Volume fraction of the vapour phase, $V_{f, V}$, at the capillary wall in the vicinity of the interface for different heat transfer coefficients in four time moments.
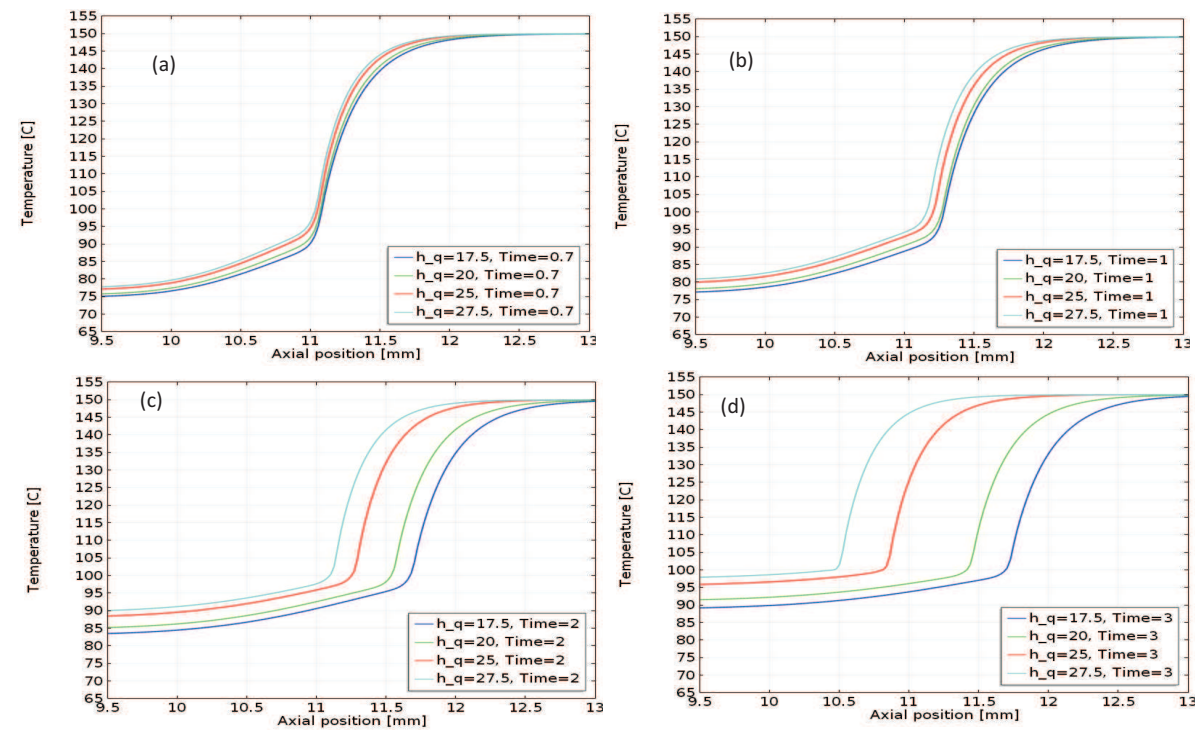

Figure 11: Temperature at the capillary wall for different heat transfer coefficients in four time instants. 


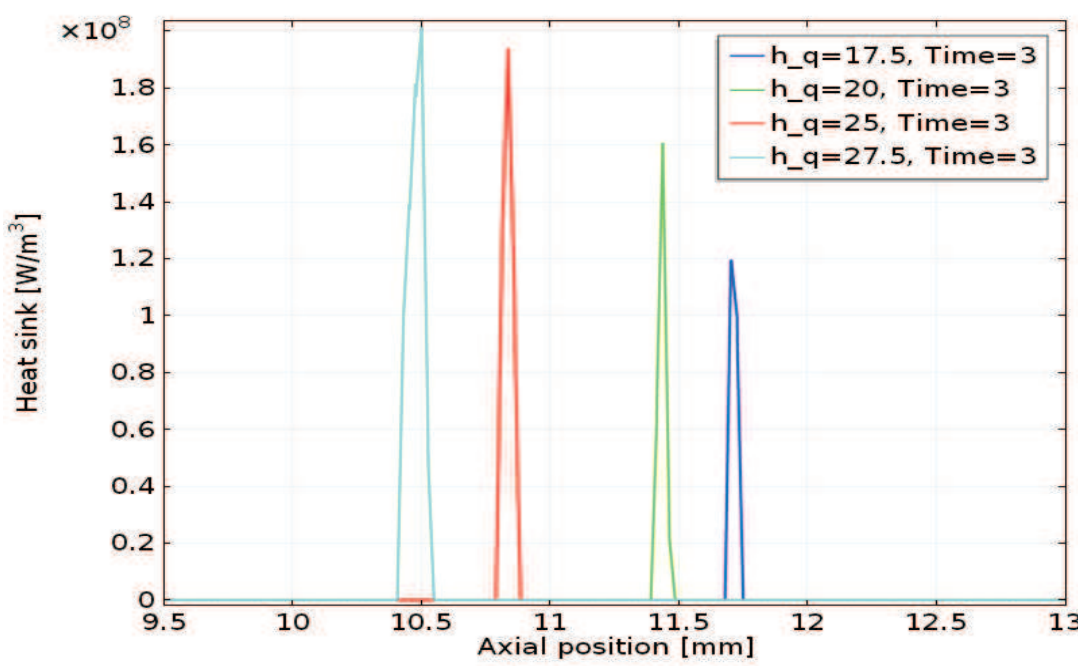

Figure 12: The heat sink, $Q_{\operatorname{sink}}$, at the capillary wall for different heat transfer coefficients for $t=3 \mathrm{~s}$.
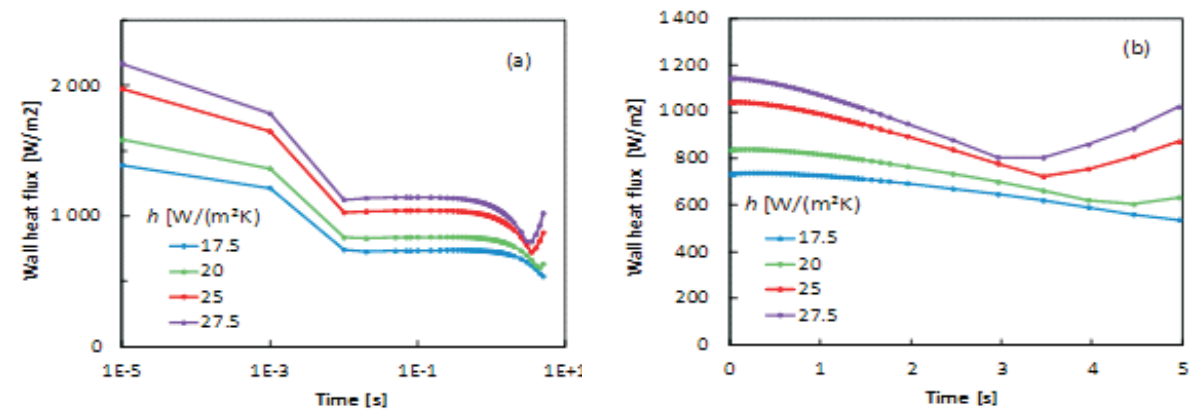

Figure 13: The heat sink, $Q_{\operatorname{sink}}$, at the capillary wall for different heat transfer coefficients for $t=3 \mathrm{~s}$.

\subsection{Fluid superheating and limitations of the model}

The presented model can be applied when there is no liquid superheating or the superheating is relatively low. The effect of superheating depends on the heat delivered to the capillary and appears when the time of heating is long enough. Figure 17 presents temperature at the capillary wall at $t=3.5 \mathrm{~s}$ for four values of the heat transfer coefficient $h$. It is seen that for $h=25$ and $27.5 \mathrm{~W} /\left(\mathrm{m}^{2} \mathrm{~K}\right)$ the fluid temperature below the phase interface is above the saturation temperature with the minimum equal to 


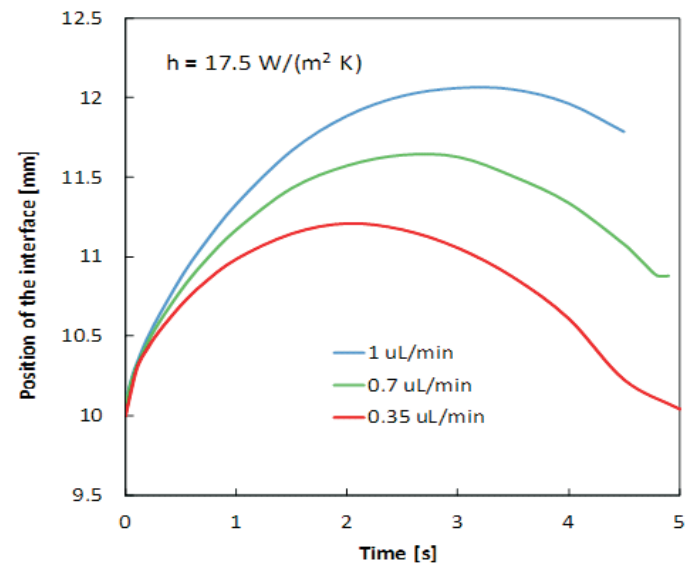

Figure 14: Time dependence of the interface position for different mass flow rates.
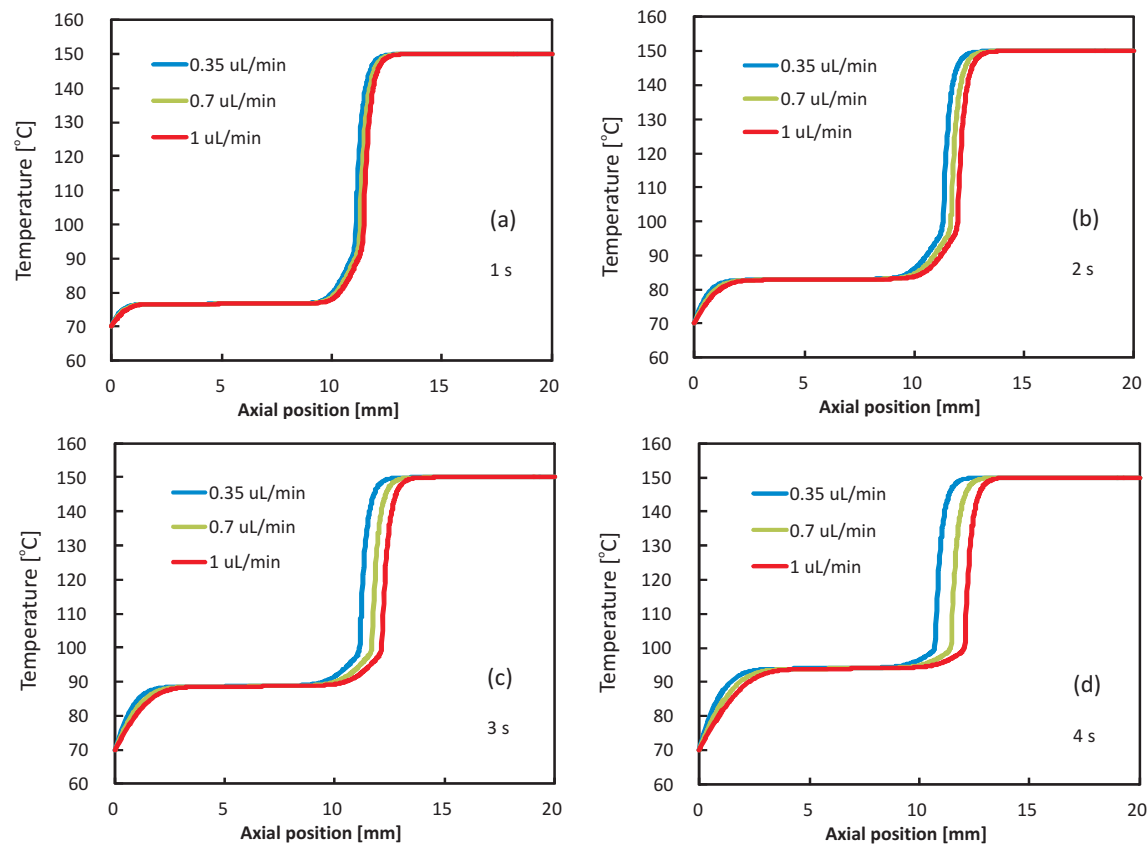

Figure 15: Temperature at the capillary wall for different mass flow rates: a) $t=1 \mathrm{~s}$, (b) $t=2 \mathrm{~s}$, (c) $t=3 \mathrm{~s}$, (d) $t=4 \mathrm{~s}$.

the saturation temperature close to the interface. Such phenomenon is not properly depicted in the model. As can be noticed when the superheating 

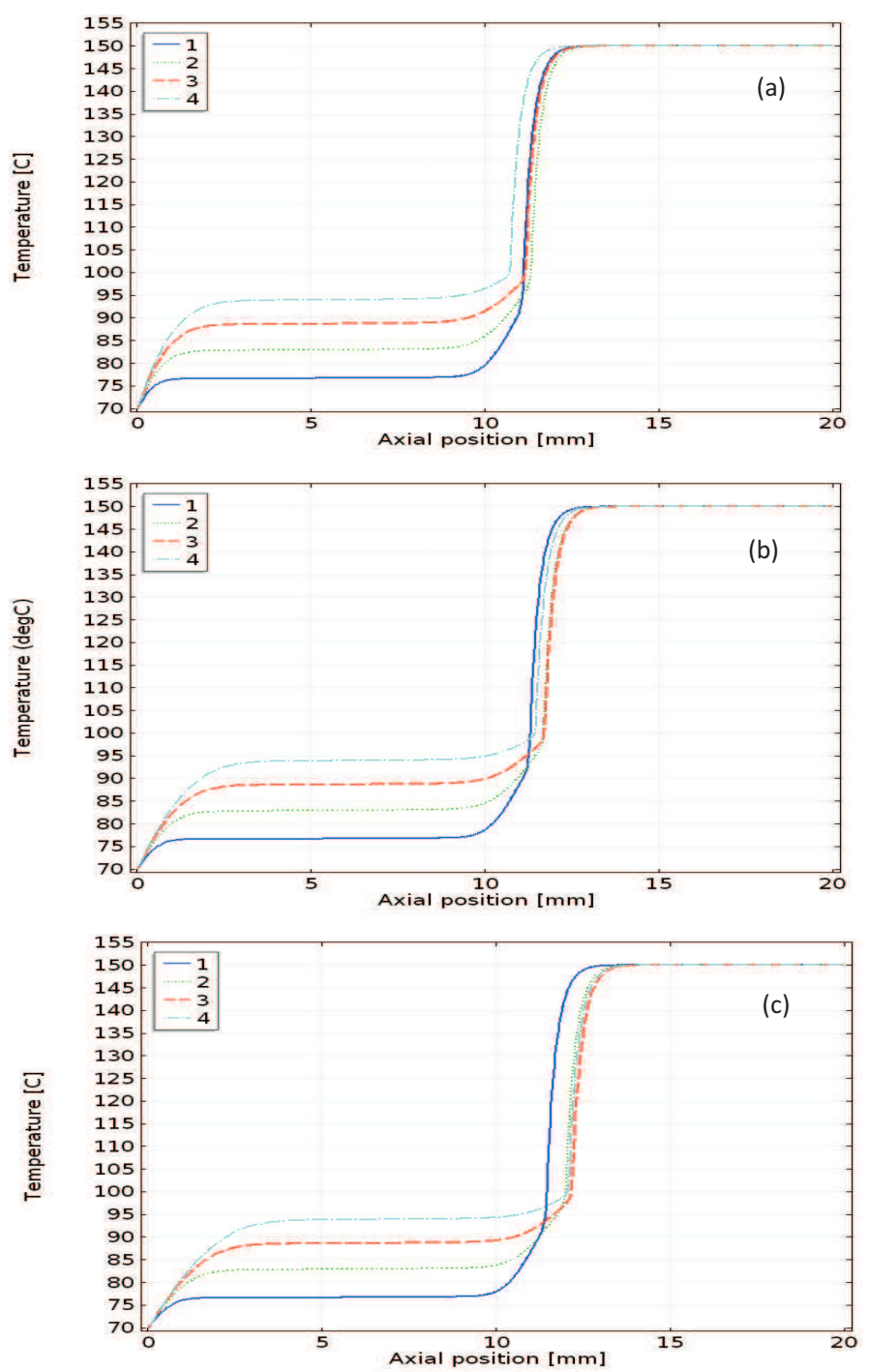

Figure 16: Temperature at the capillary wall in different time moments for different mass flow rates: (a) $Q_{m}=0.35 \mu \mathrm{L} / \mathrm{min}$, (b) $Q_{m}=0.7 \mu \mathrm{L} / \mathrm{min}$, (c) $Q_{m}=1 \mu \mathrm{L} / \mathrm{min}$. The numbers of legend present time in seconds.

increases, the interface position starts moving up, which seems against the expectations.

The phenomenon of superheating and its influence can be analysed using the results presented in Fig. 18, where it is shown how temperature, evap- 


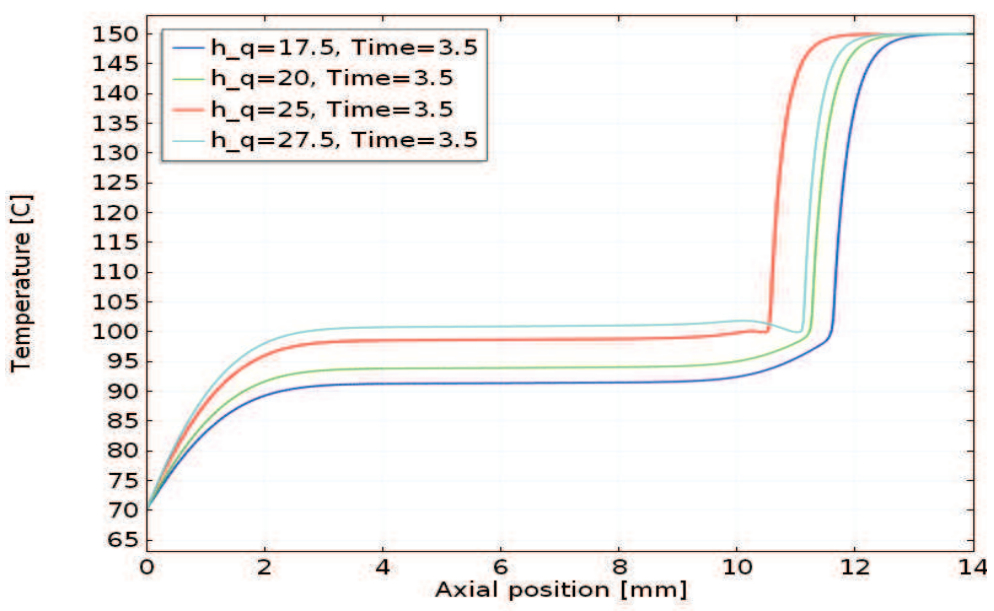

Figure 17: Temperature of the capillary wall at time $t=3.5 \mathrm{~s}$ for different values of heat transfer coefficient $h$.

oration rate, $\dot{m}$, and the smoothed representation of the phase interface between the two phases $\delta$ changes in the vicinity of the phase interface. The product of $\dot{m}$ and $\delta$ plays an important role in the model equations (see Eqs. (13), (19) and (20)).

For $t=0.9 \mathrm{~s}$ the fluid temperature exceeds the saturation temperature only for vapour phase, i.e., above the phase interface, where $\delta$ is equal to zero. It means that the product $\dot{m} \delta=0$ everywhere, so there is no evaporation. The evaporations starts at about $t=1 \mathrm{~s}$ when there can be observed a region where $\dot{m} \delta>0$. This region is at the upper boundary of the phase interface. For the next $3 \mathrm{~s}$ the temperature in the phase interface region (i.e., in the region where $\delta>0$ ) increases, so does the product $\dot{m} \delta$, because the overlapping of the phase interface region and the region where $\dot{m}>0$ is bigger. For $t=4.85 \mathrm{~s}$ the temperature is equal to the saturation temperature in the whole phase interface region. Since that moment a region of superheated liquid can be observed; it is at the lower boundary of the phase interface region. The temperature of the superheated liquid increases with time. This behaviour is in agreement with the expectations. The problem arises however when position of the phase interface region is analysed. Before the evaporation starts, the phase interface moves up (because imposed inflow and capillary forces exceed the gravitational force). When evaporation starts the phase interface moves up slowly. When the liquid loss due to evaporation is big enough, the phase interface starts moving down. This be- 

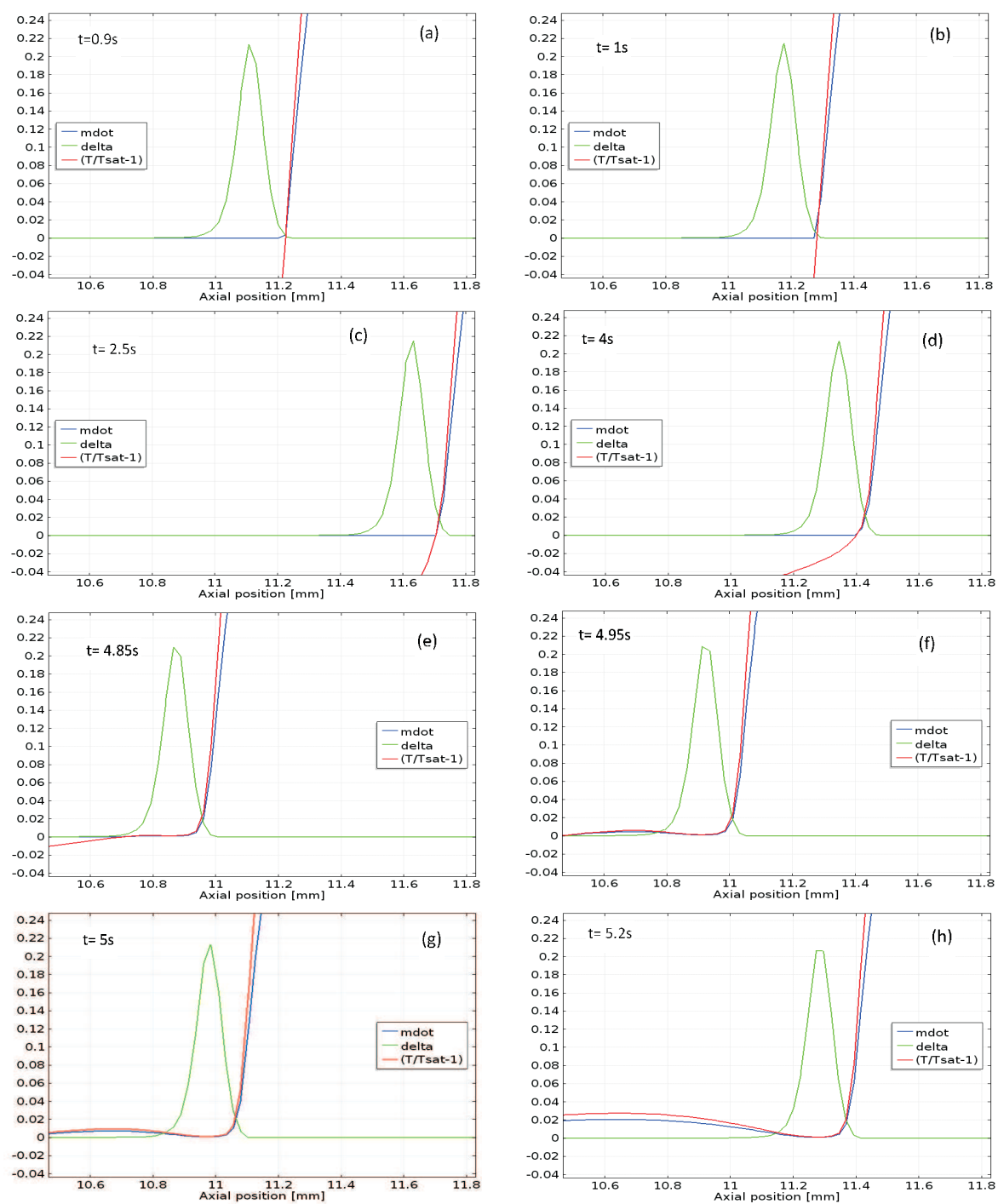

Figure 18: Evaporation rate $\dot{m}$, smoothed representation of the interface between the two phases $\delta$ and temperature in the vicinity of the phase interface in several time moments for mass flow rate $0.7 \mu \mathrm{L} / \mathrm{min}$ and heat transfer coefficient $\left.17.5 \mathrm{~W} / \mathrm{m}^{2} \mathrm{~K}\right)$. All quantities are expressed in arbitrary units.

haviour is in agreement with the expectations. However when superheating effect is strongly expressed, the movement of the phase interface changes and the phase interface moves up again, which is against the anticipations. 
One of the reasons of this behaviour seen in the model is the evaluation of the mass flux leaving the interface. This evaluation, given as Eq. (12), assumes that there is only one phase interface from which evaporation can take place. When liquid superheating occurs this definition needs a modification, which should be a goal of a further analysis.

\section{Conclusions}

The simulations of capillary pumping were performed for water in a capillary of internal diameter of $0.2 \mathrm{~mm}$. The liquid temperature at the capillary inlet was set to $70^{\circ} \mathrm{C}$. The fluid inside the capillary was heated through the wall, which external temperature was $150^{\circ} \mathrm{C}$.

In the simulations, after the initial build up, the position of the meniscus in the capillary is constant. The location of the meniscus depends on the heat flux on the capillary wall, which was controlled by changing the heat transfer coefficient, $h$. With the increasing $h$, the liquid reaches the saturation temperature over a shorter wall height and the meniscus forms closer to the capillary inlet. Increasing the inlet flow rate shifts the meniscus further from the capillary inlet. The pressure jump at the meniscus surface was also calculated and for $h=17.5 \mathrm{~W} / \mathrm{m}^{2} \mathrm{~K}$ it equals to $450 \mathrm{~Pa}$.

Acknowledgement This work was supported by Polish state budget for scientific research in the years $2010-2013$ as a research project N N513 334738 .

Received 10 December 2014

\section{References}

[1] Serin V., Médéeic B., Lavieille P., Miscevic M.: Heat and mass transfer in capillary-pumped liquid films in square cross-section minichannels. Heat Transfer Eng. 29(2008), 11, 913-923.

[2] Yarin L.P., Mosyak A., Hetsroni G.: Fluid Flow, Heat Transfer and Boiling in Microchannels. Springer, Berlin 2009.

[3] COMSOL CFD Module User's Guide: Theory for the Two-Phase Flow Interfaces. Version: COMSOL 4.1, Oct. 2010.

[4] COMSOL CFD Module User's Guide: Theory for the Phase Field Interface. Version: COMSOL 4.1, Oct. 2010. 